\title{
MEROMORPHIC FUNCTIONS SHARING A SINGLE VALUE WITH UNIT WEIGHT
}

\author{
INDRAJIT LAHIRI AND Nintu MANDAL
}

\begin{abstract}
We prove a uniqueness theorem for meromorphic functions sharing a single value with unit weight which improves a recent result of A. H. H. Al-Khaladi.
\end{abstract}

\section{Introduction, definitions and results}

Let $f$ and $g$ be two nonconstant meromorphic functions defined in the open complex plane C. For $a \in \mathbf{C} \cup\{\infty\}$ we say that $f$ and $g$ share the value $a \mathbf{C M}$ (counting multiplicities) if the $a$-points of $f$ and $g$ coincide in locations and multiplicities. If we do not consider the multiplicities, we say that $f$ and $g$ share the value $a$ IM (ignoring multiplicites). Though for the standard definitions and notations of the value distribution theory we refer to [3], some definitions and notations are given in the paper.

Definition 1.1 [6]. Let $m$ be a positive integer. We denote by $N(r, a ; f \mid \leq m) \quad(N(r, a ; f \mid \geq m))$ the counting function of those a-points of $f$ whose multiplicities are not greater (less) than $m$, where each a-point is counted according to its multiplicity.

In a like manner we define $N(r, a ; f \mid<m)$ and $N(r, a ; f \mid>m)$.

Also $\bar{N}(r, a ; f \mid \leq m), \bar{N}(r, a ; f \mid \geq m), \bar{N}(r, a ; f \mid<m)$ and $\bar{N}(r, a ; f \mid>m)$ are defined similarly where in counting the a-points of $f$ we ignore the multiplicities.

Further we agree to take $\bar{N}(r, a ; f \mid \leq \infty)=\bar{N}(r, a ; f)$ and $N(r, a ; f \mid \leq \infty)=$ $N(r, a ; f)$.

Finally we define $N_{2}(r, a ; f)=\bar{N}(r, a ; f)+\bar{N}(r, a ; f \mid \geq 2)$.

In [8] R. Nevanlinna proved the following theorem.

THEOREM A [8]. Let $f$ and $g$ be two nonconstant entire functions satisfying $N(r, 0 ; f) \equiv N(r, 0 ; g) \equiv 0$. If $f$ and $g$ share the value $1 C M$ then either $f \equiv g$ or $f g \equiv 1$.

2000 Mathematics Subject Classification. 30D35.

Key words and phrases. Meromorphic functions, Uniqueness, Weighted sharing.

Received June 1, 2005; revised July 26, 2005. 
Recently Al-khaladi [1] improved Theorem A and proved the following result.

THEOREM B [1]. Let $f$ and $g$ be two nonconstant meromorphic functions satisfying $\bar{N}(r, 0 ; g)+\bar{N}(r, \infty ; g)=S(r, g)$ and $\bar{N}(r, \infty ; f)=S(r, f)$. If $f$ and $g$ share the value $1 C M$ then $f$ and $g$ satisfy one of the following:

(i) $f-1 \equiv c(g-1)$, where $c$ is a nonzero constant. In particular, if $c=1$ then $f \equiv g$;

(ii) $(f-b) g \equiv 1-b$, where $(b \neq 1)$ is a constant. In particular, if $b=0$ then $f g \equiv 1$;

(iii) $T(r, f)=N(r, 0 ; f \mid \leq 2)+S(r, f)$ and $T(r, g)=N\left(r, 0 ; f^{\prime} \mid \leq 1\right)+S(r, f)$.

R. Brück [2] proved the following result involving a nonconstant entire function and its derivative.

THEOREM C [2]. Let $f$ be a nonconstant entire function satisfying $N\left(r, 0 ; f^{\prime}\right)$ $=S(r, f)$. If $f$ and $f^{\prime}$ share the value $1 C M$ then $f-1 \equiv c(g-1)$, where $c$ is a nonzero constant.

As a consequence of Theorem B Al-khaladi [1] improved Theorem C and proved the following result.

THEOREM D [1]. Let $f$ be a nonconstant meromorphic function satisfying $\bar{N}\left(r, 0 ; f^{\prime}\right)+\bar{N}(r, \infty ; f)=S(r, f)$. If $f$ and $f^{(k)}(k \geq 1)$ share the value $1 C M$ then $f-1 \equiv c\left(f^{(k)}-1\right)$, where $c$ is a nonzero constant.

However a better result than Theorem $\mathrm{D}$ is proved in [7]. Considering $f(z)=\left(e^{z}-1\right)\left(e^{z}+1\right)^{2}+1$ and $g(z)=e^{z}$ Al-khaladi pointed out that in Theorem $\mathrm{B}$ the $\mathrm{CM}$ sharing of the value 1 cannot be replaced by the sharing of simple 1-points only. Following example shows that in Theorem B it is not even possible to replace the $\mathrm{CM}$ sharing of the value 1 by IM sharing.

Example 1.1. Let $f(z)=2 e^{z}-e^{2 z}$ and $g(z)=e^{z}$. Then $\bar{N}(r, 0 ; g)=$ $\bar{N}(r, \infty ; g)=S(r, g), \bar{N}(r, \infty ; f)=S(r, f)$ and $f, g$ share 1 IM. Also we see that none of the possibilities of Theorem $B$ occurs.

So it is a natural query to explore the possibility of relaxing the nature of sharing the value 1 in Theorem $\mathrm{B}$. The notion of weighted sharing of values renders a useful tool for this purpose. In the following definition we explain this idea, which measures how close a shared value is to being shared IM or to being shared CM.

DeFINITION $1.2[4,5]$. Let $k$ be a nonnegative integer or infinity. For $a \in \mathbf{C} \cup\{\infty\}$ we denote by $E_{k}(a ; f)$ the set of all a-points of $f$ where an a-point of multiplicity $m$ is counted $m$ times if $m \leq k$ and $k+1$ times if $m>k$. If $E_{k}(a ; f)=E_{k}(a ; g)$, we say that $f, g$ share the value $a$ with weight $k$. 
The definition implies that if $f, g$ share a value $a$ with weight $k$ then $z_{o}$ is a zero of $f-a$ with multiplicity $m(\leq k)$ if and only if it is a zero of $g-a$ with multiplicity $m(\leq k)$ and $z_{o}$ is a zero of $f-a$ with multiplicity $m(>k)$ if and only if it is a zero of $g-a$ with multiplicity $n(>k)$ where $m$ is not necessarily equal to $n$.

We write $f, g$ share $(a, k)$ to mean that $f, g$ share the value $a$ with weight $k$. Clearly if $f, g$ share $(a, k)$ then $f, g$ share $(a, p)$ for all integers $p, 0 \leq p<k$. Also we note that $f, g$ share a value $a$ IM or CM if and only if $f, g$ share $(a, 0)$ or $(a, \infty)$ respectively.

Following theorem is the main result of the paper.

THEOREM 1.1. Let $f$ and $g$ be two nonconstant meromorphic functions such that $\bar{N}(r, 0 ; g)+\bar{N}(r, \infty ; g)=S(r, g), \quad N(r, 0 ; f)+N_{2}(r, \infty ; f) \leq T(r, f)+S(r, f)$ and $\bar{N}(r, \infty ; f) \leq \lambda T(r, f)+S(r, f)$ for a constant $\lambda(0<\lambda<1)$. If $f$, g share $(1,1)$ then $f$ and $g$ satisfy one of the following:

(i) $f-1 \equiv c(g-1)$, where $c$ is a nonzero constant. In particular, if $c=1$ then $f \equiv g$;

(ii) $(f-b) g \equiv 1-b$, where $(b \neq 1)$ is a constant. In particular, if $b=0$ then $f g \equiv 1$;

(iii) $T(r, f)=N(r, 0 ; f \mid \leq 2)+N_{2}(r, \infty ; f)+S(r, f), N\left(r, 0 ; f^{\prime} \mid \leq 1\right) \leq T(r, g)$ $+\bar{N}(r, \infty ; f)+S(r, f)$ and $T(r, g) \leq N\left(r, 0 ; f^{\prime} \mid \leq 1\right)+\bar{N}(r, \infty ; f \mid \geq 2)+$ $S(r, f)$.

Following example shows that the condition $N(r, 0 ; f)+N_{2}(r, \infty ; f) \leq$ $T(r, f)+S(r, f)$ is necessary for Theorem 1.1 .

Example 1.2. Let $f(z)=\frac{2-e^{2 z}}{2-e^{z}}$ and $g(z)=e^{z}$. Then $f, g$ share $(1, \infty)$ and $\bar{N}(r, 0 ; g)+\bar{N}(r, \infty ; g)=S(r, g) . \quad$ Also $\quad \bar{N}(r, \infty ; f)=N_{2}(r, \infty ; f)=N\left(r, 2 ; e^{z}\right)=$ $\frac{1}{2} T(r, f)+S(r, f)$ and $N(r, 0 ; f \mid \leq 2)=N(r, 0 ; f)=N\left(r, 2 ; e^{2 z}\right)=T(r, f)+S(r, f)$. Further we see that none of the possibilities of Theorem 1.1 holds.

Following example shows that for Theorem 1.1 the condition $\bar{N}(r, \infty ; f) \leq$ $\lambda T(r, f)+S(r, f)$ is necessary, where $0<\lambda<1$.

Example 1.3. Let $f(z)=\frac{2}{1+e^{z}}$ and $g(z)=e^{z}$. Then $f, g$ share $(1, \infty)$ and $\bar{N}(r, 0 ; g)+\bar{N}(r, \infty ; g)=S(r, g)$. Also $\quad N(r, 0 ; f)=S(r, f), \quad \bar{N}(r, \infty ; f)=$ $N_{2}(r, \infty ; f)=T(r, f)+S(r, f)$ and $\bar{N}(r, \infty ; f \mid \geq 2) \equiv 0$. Further none of the possibilities of Theorem 1.1 occurs.

Following example shows that the condition $\bar{N}(r, 0 ; g)=S(r, g)$ is necessary for Theorem 1.1. 
Example 1.4. Let $f(z)=e^{z}-1$ and $g(z)=\left(e^{z}-1\right)^{2}$. Then $f, g$ share $(1, \infty), \bar{N}(r, \infty ; f)=S(r, f), \bar{N}(r, \infty ; g)=S(r, g)$ and $\bar{N}(r, 0 ; g) \neq S(r, g)$. Also we see that none of the possibilities of Theorem 1.1 holds.

Following example shows that the condition $\bar{N}(r, \infty ; g)=S(r, g)$ is necessary for Theorem 1.1.

Example 1.5. Let $f(z)=1+e^{2 z}$ and $g(z)=\frac{1}{1-e^{z}}$. Then $f, g$ share $(1, \infty)$, $\bar{N}(r, \infty ; f)=S(r, f), \bar{N}(r, 0 ; g)=S(r, g)$ and $\bar{N}(r, \infty ; g) \neq S(r, g)$. Also none of the possibilities of Theorem 1.1 occurs.

Also Example 1.1 shows that in Theorem 1.1 it is not possible to relax the nature sharing from $(1,1)$ to $(1,0)$.

Finally following three examples show that all the three possibilities of Theorem 1.1 can actually occur.

Example 1.6. Let $f(z)=3 e^{z}-2$ and $g(z)=e^{z}$. Then $f, g$ share $(1, \infty)$, $\bar{N}(r, 0 ; g)+\bar{N}(r, \infty ; g)=S(r, g)$ and $\bar{N}(r, \infty ; f)=S(r, f)$. Also $f-1 \equiv 3(g-1)$, which is the possibility (i) of Theorem 1.1.

Example 1.7. Let $f(z)=2-\frac{1}{e^{z}}$ and $g(z)=e^{z}$. Then $f, g$ share $(1, \infty)$, $\bar{N}(r, 0 ; g)+\bar{N}(r, \infty ; g)=S(r, g)$ and $\bar{N}(r, \infty ; f)=S(r, f) . \quad$ Also $(f-2) g \equiv 1-2$, which is the possibility (ii) of Theorem 1.1.

Example 1.8. Let $f(z)=\frac{e^{z}\left(1+e^{z}\right)}{e^{z}-1}$ and $g(z)=-e^{2 z}$. Then $f, g$ share $(1, \infty), \quad T(r, f)=T(r, g)+O(1), \quad N(r, 0 ; f)=N(r, 0 ; f \mid \leq 2)=N\left(r,-1 ; e^{z}\right)=$ $\frac{1}{2} T(r, f)+S(r, f), \quad \bar{N}(r, \infty ; f)=N_{2}(r, \infty ; f)=N\left(r, 1 ; e^{z}\right)=\frac{1}{2} T(r, f)+S(r, f)$, $\bar{N}(r, \infty ; f \mid \geq 2) \equiv 0$ and $\bar{N}(r, 0 ; g)+\bar{N}(r, \infty ; g)=S(r, g)$. Since

$$
\begin{aligned}
N\left(r, 0 ; f^{\prime} \mid \leq 1\right) & =N\left(r, 1+\sqrt{2} ; e^{z}\right)+N\left(r, 1-\sqrt{2} ; e^{z}\right) \\
& =2 T\left(r, e^{z}\right)+S\left(r, e^{z}\right) \\
& =T(r, g)+S(r, f),
\end{aligned}
$$

it follows that $T(r, f)=N(r, 0 ; f \mid \leq 2)+N_{2}(r, \infty ; f)+S(r, f), \quad N\left(r, 0 ; f^{\prime} \mid \leq 1\right)$ $\leq T(r, g)+\bar{N}(r, \infty ; f)+S(r, f)$ and $T(r, g) \leq N\left(r, 0 ; f^{\prime} \mid \leq 1\right)+\bar{N}(r, \infty ; f \mid \geq 2)+$ $S(r, f)$, which is the possibility (iii) of Theorem 1.1 .

Following result is a direct consequence of Theorem 1.1 and improves Theorem B. 
COROLlary 1.1. Let $f$ and $g$ be two nonconstant meromorphic functions satisfying $\bar{N}(r, 0 ; g)+\bar{N}(r, \infty ; g)=S(r, g)$ and $\bar{N}(r, \infty ; f)=S(r, f)$. If $f$ and $g$ share $(1,1)$ then $f$ and $g$ satisfy one of the following:

(i) $f-1 \equiv c(g-1)$, where $c$ is a nonzero constant. In particular, if $c=1$ then $f \equiv g$;

(ii) $(f-b) g \equiv 1-b$, where $(b \neq 1)$ is a constant. In particular, if $b=0$ then $f g \equiv 1$;

(iii) $T(r, f)=N(r, 0 ; f \mid \leq 2)+S(r, f)$ and $T(r, g)=N\left(r, 0 ; f^{\prime} \mid \leq 1\right)+S(r, f)$.

We now explain some more notations.

DEFINITION 1.3 [5]. Let $f$ and $g$ share a value a IM. We denote by $\bar{N}_{*}(r, a ; f, g)$ the counting function of those a-points of $f$ whose multiplicities are not equal to the multriplicities of the corresponding a-points of $g$, where each a-point is counted only once.

Clearly $\bar{N}_{*}(r, a ; f, g) \equiv \bar{N}_{*}(r, a ; g, f)$.

DeFINITION 1.4. We denote by $N_{0}\left(r, 0 ; f^{(k)}\right)\left(\bar{N}_{0}\left(r, 0 ; f^{(k)}\right)\right)$ the counting function (reduced counting function) of those zeros of $f^{(k)}$ which are not the zeros of $f$.

DEFINITION 1.5. We denote by $N_{\otimes}\left(r, 0 ; f^{(k)}\right)\left(\bar{N}_{\otimes}\left(r, 0 ; f^{(k)}\right)\right)$ the counting function (reduced counting function) of those zeros of $f^{(k)}$ which are not the zeros of $f(f-1)$.

DEFINITION 1.6. We denote by $N_{\oplus}\left(r, 0 ; f^{(k)}\right)\left(\bar{N}_{\oplus}\left(r, 0 ; f^{(k)}\right)\right)$ the counting function (reduced counting function) of those zeros of $f^{(k)}$ which are not the zeros of $f-1$.

Throughout the paper we mean by $f, g$ two nonconstant meromorphic functions defined in the open complex plane $\mathbf{C}$.

\section{Lemmas}

In this section we present some necessary lemmas. Henceforth we denote by $H$ the function defined by

$$
H=\left(\frac{f^{\prime \prime}}{f^{\prime}}-\frac{2 f^{\prime}}{f-1}\right)-\left(\frac{g^{\prime \prime}}{g^{\prime}}-\frac{2 g^{\prime}}{g-1}\right) .
$$

Lemma 2.1 [5]. If $f, g$ share $(1,1)$ and $H \not \equiv 0$ then 
(i) $N(r, 1 ; f \mid \leq 1) \leq N(r, H)+S(r, f)+S(r, g)$,

(ii) $N(r, 1 ; g \mid \leq 1) \leq N(r, H)+S(r, f)+S(r, g)$.

Lemma $2.2[5]$. Let $f, g$ share $(1,0)$ and $H \not \equiv 0$. Then

$$
\begin{aligned}
N(r, H) \leq & \bar{N}(r, \infty ; f \mid \geq 2)+\bar{N}(r, 0 ; f \mid \geq 2)+\bar{N}(r, \infty ; g \mid \geq 2)+\bar{N}(r, 0 ; g \mid \geq 2) \\
& +\bar{N}_{*}(r, 1 ; f, g)+\bar{N}_{\otimes}\left(r, 0 ; f^{\prime}\right)+\bar{N}_{\otimes}\left(r, 0 ; g^{\prime}\right) .
\end{aligned}
$$

Lemma 2.3 [6]. If $k$ is a positive integer then

$$
N_{0}\left(r, 0 ; f^{(k)}\right) \leq k \bar{N}(r, \infty ; f)+N(r, 0 ; f \mid<k)+k \bar{N}(r, 0 ; f \mid \geq k)+S(r, f) .
$$

LEMMA 2.4. If $f, g$ share $(1,1)$ then

$$
\bar{N}_{0}\left(r, 0 ; g^{\prime}\right)+\bar{N}(r, 1 ; g \mid \geq 2)+\bar{N}_{*}(r, 1 ; f, g) \leq 3 \bar{N}(r, 0 ; g)+3 \bar{N}(r, \infty ; g)+S(r, g) .
$$

Proof. Since $f, g$ share $(1,1)$, we get by Lemma 2.3 for $k=1$

$$
\begin{aligned}
\bar{N}_{0}(r & \left., 0 ; g^{\prime}\right)+\bar{N}(r, 1 ; g \mid \geq 2)+\bar{N}_{*}(r, 1 ; f, g) \\
& \leq \bar{N}_{0}\left(r, 0 ; g^{\prime}\right)+2 \bar{N}(r, 1 ; g \mid \geq 2) \\
& \leq 3 N_{0}\left(r, 0 ; g^{\prime}\right) \\
& \leq 3 \bar{N}(r, 0 ; g)+3 \bar{N}(r, \infty ; g)+S(r, g) .
\end{aligned}
$$

This proves the lemma.

\section{Proof of the main result}

Proof of Theorem 1.1. We consider the following two cases.

Case I. Let $H \equiv 0$. Then on integration we get

$$
f-1 \equiv \frac{g-1}{A-B(g-1)},
$$

where $A(\neq 0)$ and $B$ are constants.

If $B=0$ then from (3.1) we get

$$
f-1 \equiv c(g-1),
$$

where $c=\frac{1}{A}$ is a nonzero constant. This is possibility (i) of the theorem. theorem

Let $B \neq 0$. If $A+B \neq 0$ then from (3.1) we get by the second fundamental 


$$
\begin{aligned}
T(r, g) & \leq \bar{N}(r, 0 ; g)+\bar{N}(r, \infty ; g)+\bar{N}\left(r, \frac{A+B}{B} ; g\right)+S(r, g) \\
& =\bar{N}(r, \infty ; f)+S(r, g) \\
& \leq \lambda T(r, f)+S(r, g) \\
& =\lambda T(r, g)+S(r, g),
\end{aligned}
$$

which is a contradiction as $0<\lambda<1$.

Therefore $A+B=0$ and so from (3.1) we get

$$
\left(f-\frac{B-1}{B}\right) g \equiv \frac{1}{B} \text {. }
$$

If we put $b=\frac{B-1}{B}$ then $b \neq 1$ and from above we get

$$
(f-b) g \equiv 1-b,
$$

which is possibility (ii) of the theorem.

CASE II. Let $H \not \equiv 0$. Since $f, g$ share $(1,1)$, by the second fundamental theorem we get

$$
\begin{aligned}
T(r, g) & \leq \bar{N}(r, 0 ; g)+\bar{N}(r, \infty ; g)+\bar{N}(r, 1 ; g)+S(r, g) \\
& =\bar{N}(r, 1 ; g)+S(r, g) \\
& \leq T(r, f)+S(r, g) .
\end{aligned}
$$

This shows that every $S(r, g)$ is replacable by $S(r, f)$. Let $h=(f-1) /(g-1)$. Since $f, g$ share $(1,1)$ we get by Lemma 2.4

$$
\begin{aligned}
\bar{N}(r, 0 ; h) & \leq \bar{N}_{*}(r, 1 ; f, g)+\bar{N}(r, \infty ; g) \\
& \leq 3 \bar{N}(r, 0 ; g)+4 \bar{N}(r, \infty ; g) \\
& =S(r, g) \\
& =S(r, f)
\end{aligned}
$$

and

$$
\begin{aligned}
\bar{N}(r, \infty ; h) & \leq \bar{N}_{*}(r, 1 ; f, g)+\bar{N}(r, \infty ; f) \\
& \leq 3 \bar{N}(r, 0 ; g)+3 \bar{N}(r, \infty ; g)+\bar{N}(r, \infty ; f) \\
& =\bar{N}(r, \infty ; f)+S(r, g) \\
& =\bar{N}(r, \infty ; f)+S(r, f) .
\end{aligned}
$$

Since

$$
f^{\prime}=h(g-1)\left(\frac{h^{\prime}}{h}+\frac{g^{\prime}}{g-1}\right)
$$


we see that possible zeros of $f^{\prime}$ occur from the following sources: (i) zeros of $h$, (ii) zeros of $g-1$ and (ii) zeros of $\frac{h^{\prime}}{h}+\frac{g^{\prime}}{g-1}$.

Let $z_{0}$ be a simple zero of $g-1$. Since $f, g$ share $(1,1), z_{0}$ is neither a zero nor a pole of $h$. On the other hand $z_{0}$ is a simple pole of $\frac{h^{\prime}}{h}+\frac{g^{\prime}}{g-1}$. Hence $z_{0}$
is not a zero of $f^{\prime}$. Therefore by Lemma 2.4 we get

$$
\begin{aligned}
\bar{N}(r, & \left.0 ; f^{\prime}\right) \\
& \leq \bar{N}(r, 0 ; h)+\bar{N}(r, 1 ; g \mid \geq 2)+T\left(r, \frac{h^{\prime}}{h}+\frac{g^{\prime}}{g-1}\right) \\
& \leq 3 \bar{N}(r, 0 ; g)+3 \bar{N}(r, \infty ; g)+N\left(r, \frac{h^{\prime}}{h}\right)+N\left(r, \frac{g^{\prime}}{g-1}\right)+S(r, f) \\
& \leq \bar{N}(r, 0 ; h)+\bar{N}(r, \infty ; h)+\bar{N}(r, 1 ; g)+\bar{N}(r, \infty ; g)+S(r, f) \\
& \leq N(r, 1 ; g \mid \leq 1)+\bar{N}(r, 1 ; g \mid \geq 2)+\bar{N}(r, \infty ; f)+S(r, f) \\
& \leq N(r, 1 ; g \mid \leq 1)+3 \bar{N}(r, 0 ; g)+3 \bar{N}(r, \infty ; g)+\bar{N}(r, \infty ; f)+S(r, f) \\
& =N(r, 1 ; g \mid \leq 1)+\bar{N}(r, \infty ; f)+S(r, f) .
\end{aligned}
$$
get

$$
\begin{aligned}
N(r, 1 ; g \mid \leq 1) \leq & \bar{N}(r, 0 ; f \mid \geq 2)+\bar{N}(r, 0 ; g \mid \geq 2) \\
& +\bar{N}_{*}(r, 1 ; f, g)+\bar{N}(r, \infty ; g \mid \geq 2) \\
& +\bar{N}_{\otimes}\left(r, 0 ; f^{\prime}\right)+\bar{N}_{\otimes}\left(r, 0 ; g^{\prime}\right)+\bar{N}(r, \infty ; f \mid \geq 2) \\
\leq & \bar{N}(r, 0 ; f \mid \geq 2)+\bar{N}(r, 1 ; f \mid \geq 2)+\bar{N}_{\otimes}\left(r, 0 ; f^{\prime}\right) \\
& +N_{0}\left(r, 0 ; g^{\prime}\right)+\bar{N}(r, \infty ; f \mid \geq 2)+S(r, g) \\
\leq & \bar{N}\left(r, 0 ; f^{\prime}\right)+\bar{N}(r, \infty ; f \mid \geq 2)+S(r, f) .
\end{aligned}
$$

By the second fundamental theorem and Lemma 2.3 we get

$$
\begin{aligned}
T(r, g) & \leq \bar{N}(r, 1 ; g)+\bar{N}(r, 0 ; g)+\bar{N}(r, \infty ; g)+S(r, g) \\
& \leq N(r, 1 ; g \mid \leq 1)+N_{0}\left(r, 0 ; g^{\prime}\right)+S(r, g) \\
& \leq N(r, 1 ; g \mid \leq 1)+N_{0}\left(r, 0 ; g^{\prime}\right)+S(r, g) \\
& \leq N(r, 1 ; g \mid \leq 1)+\bar{N}(r, 0 ; g)+\bar{N}(r, \infty ; g)+S(r, g) \\
& =N(r, 1 ; g \mid \leq 1)+S(r, g)
\end{aligned}
$$

so that

$$
N(r, 1 ; g \mid \leq 1)=T(r, g)+S(r, g)=T(r, g)+S(r, f)
$$


Since $f, g$ share $(1,1)$ by Lemma 2.3 we get

$$
\begin{aligned}
\bar{N}(r, 1 ; f \mid \geq 2) & =\bar{N}(r, 1 ; g \mid \geq 2) \\
& \leq N_{0}\left(r, 0 ; g^{\prime}\right) \\
& \leq \bar{N}(r, 0 ; g)+\bar{N}(r, \infty ; g)+S(r, g) \\
& =S(r, g) \\
& =S(r, f) .
\end{aligned}
$$

Now by the second fundamental theorem we get from (3.3) and the given condition

$$
\begin{aligned}
T(r, f) \leq & \bar{N}(r, \infty ; f)+N(r, 0 ; f)+\bar{N}(r, 1 ; f)-N_{\otimes}\left(r, 0 ; f^{\prime}\right)+S(r, f) \\
= & \bar{N}(r, \infty ; f)+N(r, 0 ; f)+N(r, 1 ; g \mid \leq 1)-N_{\otimes}\left(r, 0 ; f^{\prime}\right)+S(r, f) \\
\leq & N_{2}(r, \infty ; f)+N(r, 0 ; f)+\bar{N}\left(r, 0 ; f^{\prime}\right)-N_{\otimes}\left(r, 0 ; f^{\prime}\right)+S(r, f) \\
= & N_{2}(r, \infty ; f)+N(r, 0 ; f)+\bar{N}_{\otimes}\left(r, 0 ; f^{\prime}\right)-N_{\otimes}\left(r, 0 ; f^{\prime}\right) \\
& +\bar{N}(r, 1 ; f \mid \geq 2)+S(r, f) \\
= & N(r, 0 ; f)+N_{2}(r, \infty ; f)+\bar{N}_{\otimes}\left(r, 0 ; f^{\prime}\right)-N_{\otimes}\left(r, 0 ; f^{\prime}\right)+S(r, f) \\
\leq & N_{2}(r, \infty ; f)+N(r, 0 ; f)+S(r, f) \\
\leq & T(r, f)+S(r, f) .
\end{aligned}
$$

This shows that

$$
T(r, f)=N(r, 0 ; f)+N_{2}(r, \infty ; f)+S(r, f)
$$

and

$$
N_{\otimes}\left(r, 0 ; f^{\prime}\right)-\bar{N}_{\otimes}\left(r, 0 ; f^{\prime}\right)=S(r, f) .
$$

From (3.6) we get

$$
N(r, 0 ; f \mid \geq 3) \leq 3\left\{N_{\otimes}\left(r, 0 ; f^{\prime}\right)-\bar{N}_{\otimes}\left(r, 0 ; f^{\prime}\right)\right\}=S(r, f) .
$$

Hence from (3.5) we get

$$
T(r, f)=N(r, 0 ; f \mid \leq 2)+N_{2}(r, \infty ; f)+S(r, f) .
$$

Again from (3.6) we get by Lemma 2.4

$$
\begin{aligned}
\bar{N}\left(r, 0 ; f^{\prime} \mid \geq 2\right) & \leq \bar{N}(r, 1 ; f \mid \geq 3)+2\left\{N_{\otimes}\left(r, 0 ; f^{\prime}\right)-\bar{N}_{\otimes}\left(r, 0 ; f^{\prime}\right)\right\} \\
& \leq \bar{N}(r, 1 ; f \mid \geq 2)+S(r, f) \\
& =S(r, f) .
\end{aligned}
$$

So from (3.2), (3.3) and (3.4) we obtain 


$$
N\left(r, 0 ; f^{\prime} \mid \leq 1\right) \leq T(r, g)+\bar{N}(r, \infty ; f)+S(r, f)
$$

and

$$
T(r, g) \leq N\left(r, 0 ; f^{\prime} \mid \leq 1\right)+\bar{N}(r, \infty ; f \mid \geq 2)+S(r, f) .
$$

This proves the theorem.

\section{REFERENCES}

[1] A. H. H. Al-Khaladi, On two meromorphic functions that share one value CM, Kodai Math. J. 26 (2003), 259-266.

[2] R. BRÜCK, On entire functions which share one value CM with their first derivative, Results Math. 30 (1996), 21-24.

[ 3 ] W. K. Hayman, Meromorphic Functions, The Clarendon Press, Oxford, 1964.

[4] I. LAHIRI, Weighted sharing and uniqueness of meromorphic functions, Nagoya Math. J. 161 (2001), 193-206.

[5] I. LAHIRI, Weighted value sharing and uniqueness of meromorphic functions, Complex Var. Theory Appl. 46 (2001), 241-253.

[6] I. LahiRi AND S. Dewan, Value distribution of the product of a meromorphic function and its derivative, Kodai Math. J. 26 (2003), 95-100.

[ 7 ] I. LAHIRI AND A. SARKar, Uniqueness of a meromorphic function and its derivative, JIPAM, J. Inequal. Pure Appl. Math. 5 (2004), Article 20 (electronic); http://jipam.vu.edu.au/

[8] R. Nevanlinna, Einige Eindeutigkeitssätze in der Theorie der meromorphen Funktionen, Acta Math. 48 (1926), 367-391.

Department of Mathematics

UNIVERSITY OF KALYANI

WeSt BENGAL 741235

INDIA

E-mail: ilahiri@vsnl.com, ilahiri@hotpop.com 